Огляди

DOI: 10.31793/1680-1466.2020.25-2.158

\title{
Роль молекулярных часов циркадианных ритмов в патогенезе метаболического синдрома
}

\section{И.П. Кайдашев}

Украинская медицинская стоматологическая академия, г. Полтава

\begin{abstract}
Резюме. Метаболический синдром объединяет нарушения метаболизма, приводящие к увеличению массы тела, с сопутствующим системным воспалением. Важным является выявление патогенетических связей между нарушениями обмена глюкозы, липидов, окислительным стрессом, воспалением, увеличением массы тела и молекулярными механизмами циркадианных часов. В обзоре приведены современные данные об обеспечении циркадианных ритмов (ЦР) при участии центрального и периферических пейсмекеров, их молекулярных составляющих и иерархии. Главные факторы нарушений ЦР - изменение продолжительности циклов «светтемнота», нарушение светового режима, продолжительности сна, употребление еды во второй половине дня. Нарушение ЦР вызывает снижение толерантности к глюкозе, повышение инсулинорезистентности тканей, нарушает всасывание, транспортировку и депонирование липидов в организме и приводит к накоплению излишков энергии. Такие метаболические нарушения происходят параллельно с повышением уровня системного воспаления: выработки провоспалительных хемо- и цитокинов, активации иммунных клеток. Сделан вывод, что нарушения ЦР приводят к метаболическим нарушениям и системному воспалению, что соответствует понятию «метаболического синдрома».
\end{abstract}

Ключевые слова: метаболический синдром, молекулярные часы, воспаление, обмен углеводов.

Если ты не в силах изменить свой образ жизни, то тебе никто и ничто не поможет.

Iunпократ

Сегодня нарушения метаболизма, приводящие к увеличению массы тела с сопутствующим низкоинтенсивным системным воспалением, объединяются под названием метаболический синдром (МС) [1]. МС яв-

\footnotetext{
* Адреса для листування (Correspondence): Українська медична стоматологічна академія, вул. Шевченка, 2, м. Полтава, 36011, Україна.

E-mail:zdovado@ukr.net

(c) И.П. Кайдашев
}

ляется важным предшественником развития сердечно-сосудистых и других хронических заболеваний [2, 3]. Чрезвычайно важным является выявление связей между отдельными составляющими метаболического синдрома - нарушениями обмена глюкозы и липидов, окислительным стрессом, хроническим системным воспалением, повышением массы тела и т.д. [4].

Рассматривая МС как «болезнь цивилизации», ученые предположили, что в число этиологических факторов развития такого состо- 
яния может являться изменение длительности светового дня при использовании искусственного освещения, уменьшение амплитуды температуры окружающей среды, а также изменения режима работы [5, 6]. За прошедшие годы накопилось достаточно новых сведений о циркадианных ритмах, их молекулярных механизмах и связи их возможных нарушений с развитием МС. Ниже остановимся более подробно на устройстве «циркадианных часов», их молекулярных основах, связях с регуляцией обмена веществ, массы тела, воспалением и иммунными реакциями.

\section{Циркадианные часы}

Циркадианные часы - это эволюционно высоко консервативная особенность бактерий, растений и животных, которая позволяет организмам приспосабливать физиологические процессы ко времени суток [7, 8]. Этот внутренний механизм синхронизации координирует биохимические, физиологические и поведенческие процессы для поддержания синхронности с циклами света, температуры и поступлением питательных веществ. У млекопитающих, в том числе у человека, циркадианные ритмы регулируются системой временного контроля, состоящей из основного пейсмекера в супрахиазматических ядрах (СХЯ) гипоталамуса и периферических осцилляторов, расположенных по всему организму. Независимые циркадианные осцилляторы существуют в каждой клетке любой ткани и/или органа, включая печень и сердце. СХЯ управляет и координирует периферические часы с помощью еще не определенных гуморальных и нейронных сигналов, а также косвенно, модулируя активность и питание [9].

СХЯ являются циркадианным пейсмекером мозга млекопитающих, осуществляющим регуляцию ежедневной циклической активности уровня гормонов и других физиологических процессов. Свет может приводить к фазовому сдвигу эндогенных колебаний в СХЯ, синхронизируя его деятельность с циклом день-ночь окружающей среды [10]. Несколько исследований показали, что свет является самым мощным временным фактором (Zeitgeber) для инициирования циркадианной системы млекопитающих.

У млекопитающих восприятие света происходит только в сетчатке [11], где присутствуют три различных типа фоторецепторов: колбочки, палочки и недавно открытые светочувствительные ганглионарные клетки сетчатки (ipRGC). Последние играют ключевую роль в зрении, не формирующем изображение [12], т.е. регистрирующем освещенность, а также передают световую информацию от палочек и колбочек в дополнение к их собственному меланопсин-зависимому пути и могут контролировать различные виды света, оказывающих влияние на поведение [13]. Однако, открытие «генов часов» привело к установлению факта способности циркадианных генов экспрессироваться в организме повсеместно [14]. Клеточные автономные часы являются вездесущими, и каждая клетка в организме имеет циркадианные часы [15]. Кроме центральных механизмов, в регуляции циркадианных ритмов важную роль играют и «периферические часы». Показано, что циркадианные изменения передачи болевых сигналов с участием субстанции Р нуждаются в процессах циркадианно регулируемой периферической сигнализации [16].

Логично предположить, что циркадианные ритмы тесно связаны со сном. Сон является важным регулятором многих физиологических функций, которые относятся к метаболизму. Существуют весомые доказательства, что длительность и частота сна могут быть факторами риска развития диабета. В частности, недостаточная длительность сна и/или ограничение сна, плохое качество сна связаны с повышенным риском развития диабета. Такие физиологические механизмы, как инсулинорезистентность (ИР), снижение уровня лептина и повышение грелина, воспаление и неблагоприятные поведенческие модели (увеличение объема принимаемой пищи, нарушение времени ее приема, повышенная вероятность других поведенческих факторов риска - курение, сидячий образ жизни и употребление алкоголя) предрасполагают к развитию диабета и ожирения, которое само по себе является фактором риска диабета [17].

Молекулярные механизмы циркадианных часов у млекопитающих

У млекопитающих механизм циркадианных часов является клеточно автономным и исходит от ауторегуляторной транскрипционной обратной связи (рис.). 
Огляди

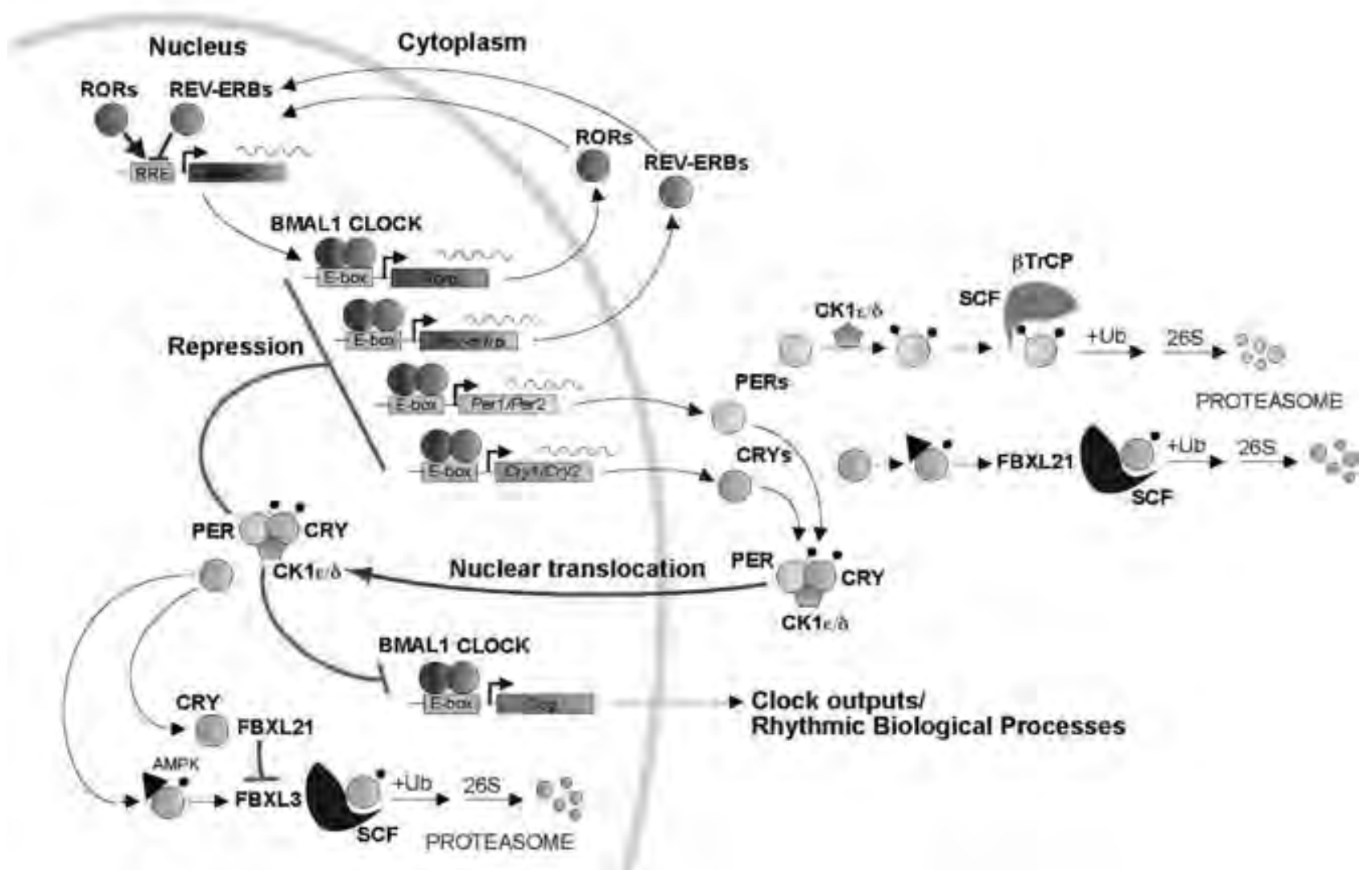

Рис. Молекулярный механизм циркадианных часов у млекопитающих [18].

В основе такой часовой системы находится ауторегуляторная транскрипционная петля обратной связи: активаторы транскрипции CLOCK (и его паралог NPAS2) и BMAL1, которые положительно регулируют экспрессию генов Period (Per1, Per2) и Cryptochrome (Cry1, Cry2) в начале временного цикла. Генные продукты Per и Cry накапливаются, димеризуются и образуют комплекс, который транслоцируется в ядро для взаимодействия с CLOCK и BMAL1, репрессируя их собственную транскрипцию. Такой цикл обратной связи занимает около 24 часов, и обмен Per и Сry тщательно регулируется Е3 убиквитин лигазным комплексом. Существуют дополнительные петли обратной связи, примыкающие к основной петле - CLOCK-BMAL1/PER-CRY. Наиболее заметной является петля, состоящая из Rev-erba (Nr1d1) и Rora, которые также прямо направлены на CLOCK-BMAL1. Эффект обратной связи инициируется транскрипцией Bmal1 (и менее выражено, Clock) и вызывает противофазные колебания BMAL1. Другие петли обратной связи включают членов семейства PAR-bZip: DBP, HLF и TEF; bZip белок, E4BP4
(Nfil3) и bHLH белки, DEC1 и DEC2 (Bhlhb2, Bhlhb3), все они являются транскрипционными мишенями CLOCK-BMAL [19].

Хотя основной «часовой» механизм является консервативным для клеток различных тканей, существуют значительные различия в относительных распространенностях индивидуальных компонентов «часов», также как и в модели настройки таких периферических часов. Такие эндогенные клеточные часы регулируют обширные ритмы генной транскрипции, в 3-10\% всей мРНК в конкретной ткани демонстрируют циркадианные ритмы в устойчивом состоянии [20]. Однако гены, которые находятся под циркадианным контролем, не перекрываются в каждой отдельной ткани, отражая необходимость временного контроля физиологического состояния клеток, свойственного для каждого уникального клеточного типа. Результатом этого является то, что циркадианные часы осуществляют широкомасштабный контроль над многими биологическими процессами, включая многие аспекты метаболизма, такие как детоксикация ксенобиотиков, гомеостаз глюкозы и липогенез [21]. 
Кроме того, сложные петли обратной связи связывают циркадианные часы с ритмичной метаболической сетью, объединяя эти системы в свет-независимую модель. Циркадианный контроль метаболизма наблюдается как на центральном, так и на местных уровнях и включает в себя часы во многих периферических тканях, в том числе в печени, поджелудочной железе, скелетных мышцах, кишечнике и жировой ткани [22]. Тонкое взаимоотношение между часами и метаболизмом является примером того, как циркадианная система интегрируется и оказывается под влиянием физиологических процессов.

Таким образом, организация циркадианной системы требует сочетания: 1) автономной иннервации периферических тканей; 2) эндокринных сигналов; 3) температуры; 4) местных сигналов. Например, симпатическая иннервация от СXЯ к PVNuk печени приводит к ежедневным ритмам уровня глюкозы в плазме преимущественно путем прямого влияния на ритм глюконеогенеза в печени [23]. Автономные пути от СХЯ передают информацию об освещенности к осцилляторам в надпочечниках и печени [23]. Симпатическая иннервация также модулирует чувствительность надпочечников к адренокортикотропному гормону (АКТГ) и прямо влияет на высвобождение глюкокортикоидов [24]. Осцилляторы в корковом и мозговом веществе надпочечников отвечают на нейрональные сигналы от СХЯ.

Среди эндокринных сигналов центральное место занимают глюкокортикоиды (ГК). Ритмичное высвобождение ГК зависит как от ритмичной продукции АКТГ и кортикотропин-рилизинг гормона, так и от входящих симпатических сигналов. Надпочечниковые часы сами по себе также осуществляют временной контроль чувствительности к АКТГиндуцированному высвобождению ГК [25].

Рассматривая вопросы, касающиеся циркадианных часов в контексте МС как «болезни цивилизации», нельзя не коснуться влияния температуры окружающей среды. В обсуждении новых концептуальных взглядов на МС обращают внимание на влияние изменения температуры окружающей среды - уменьшение диапазона колебаний вследствие кондиционирования (отопление в зимний период, охлаждение в летний) [5, 6]. Давно известно, что температура тела имеет дневные колебания, и ритм задается СХЯ. Периферические осцилляторы, включая фибробласты, печень, почки и легкие, высокочувствительны к изменению температуры [26]. Влияние температуры на периферические осцилляторы преимущественно наблюдается через транскрипционный фактор Heat shock factor (HSF1). Транскрипционная активность колеблется с циркадианным ритмом в печени и может регулироваться температурными циклами [27]. Так как HSF1 находится под влиянием многих сигнальных путей в клетке [28], температура и HSF1 могут образовывать окончательный общий путь для интеграции перенастраивающих сигналов в периферических часах.

Важными местными сигналами являются транскрипционные факторы, которые проявляют циркадианные ритмы транскрипции в печени и метаболически активных тканях [29]. Среди ритмических ядерных рецепторов находятся PPAR и члены семейств REV-ERB и ROR. Как было описано выше, ROR $\alpha$ и REV-ERB2 прямо участвуют в часовом механизме путем регулирования транскрипции BMAL1 [30], но также важны и для многих аспектов метаболической регуляции. Рецепторы ГК индуцируют транскрипцию Per и, возможно, множества других генов, контролирующих и контролируемых часами. PPAR $\alpha$, отвечающий за состояние обмена липидов и ГК, может также регулировать транскрипцию BMAL1 [31]. PPAR- $\gamma$ коактиватор-1 $\alpha$ (PGC-1 $\alpha), \quad$ транскрипционный ко-активатор обеспечивает связь между изменением состояния обмена веществ и часами. PGC-1 $\alpha$ является важным для адаптационных ответов на состояние питания и метаболизма, особенно на продолжительное голодание [32]. PGC-1 $\alpha$ является сам по себе ритмичным и активирует экспрессию Bmal-1 и Rev-erba через ко-активацию ROR [33]. PGC-1 $\alpha$ нокаутированные мыши демонстрируют разрушение множества циркадианных исходящих сигналов, включая двигательную активность, уровень потребления кислорода, экспрессию генов часов и метаболизма [33]. PGC-1 $\alpha$ также взаимодействует с сиртуином-1 (SIRT-1) NADзависимой гистоновой диацетилазой [34].

Клеточный редокс-потенциал также может служить механизмом, посредством которого состояние обмена веществ клетки воз- 
Огляди

действует на циркадианную систему. Уровни NAD отражают циркадианные колебания в печени, преимущественно благодаря транскрипционной регуляции никотинамид-фосфорибозил-трансферазы (NAmpt) через CLOCK:BMAL1 [35]. Уровни NAD также изменяются в соответствии с клеточным редокспотенциалом как следствие метаболических изменений, и это в свою очередь может прямо влиять на функцию часов.

Соотношение $\mathrm{NAD}^{+}$и NADH влияет на связывание NPAS2:BMAL1 и CLOCK: BMAL1 c DNA in vitro, предполагая один из путей, с помощью которого NAD может взаимодействовать с компонентами часов [36]. NAD ${ }^{+}$ зависимый SIRT1 также отражает ежедневные осцилляции и осуществляет обратную связь с циркадианными часами [37]. SIRT1 образует комплекс с CLOCK:BMAL1, приводя к деацетилированию PER2 и BMAL1 [38, 39].

Циркадианные часы, воспаление и иммунные реакции

СХЯ в числе других физиологических функций регулирует иммунный ответ. Известно, что смещение рабочего времени нарушает циркадианные ритмы и связано с развитием повышенной чувствительности к некоторым заболеваниям. Экспериментально установлено, что смещение рабочего времени нарушает иммунный ответ на липополисахарид (ЛПС). При этом продуцировались повышенные уровни цитокинов, таких как ФНО- $\alpha$. Эти данные показали, что циркадианная синхронизация оказывает значительное влияние на воспалительный ответ организма. Важным является то, что влияние смещения рабочего времени на воспалительный ответ предотвращалось, когда пища не давалась в рабочие часы [40].

Проведены исследования, показавшие, что существуют циркадианные изменчивости ответа иммунной системы, предполагающие участие СХЯ. Например, введение ЛПС в начале активного периода вызывало более выраженную гипертензию и синтез цитокинов, чем его введение в период отдыха. Более того, ночное введение ЛПС повышает базальную нейрональную активность, указывая на прямое воздействие воспаления на СХЯ. Двустороннее повреждение СХЯ приводило к усиленному воспалительному ответу на ЛПС [41].
Показано, что дневное кормление вызывает дисбаланс генов часов, что приводит к снижению экспрессии белков энергетического метаболизма и регуляции временных процессов. Такое несогласование ритмов периферических и центральных ритмов генов часов вследствие изменения режима кормления приводит к усиленному воспалительному ответу [42].

Многими исследованиями показано существование реципрокных взаимоотношений циркадианных ритмов и иммунной системы. Важными являются результаты исследования воздействия эндотоксина на циркадианный ритм. После периферического введения 5 мг/ кг ЛПС животным не наблюдалось изменений основных параметров циркадианной ритмичности - периодов произвольного бега амплитуды ритма, восприятия циклов свет-темнота. Перевод животных, которым вводился ЛПС, на 6-часовой цикл свет/темнота приводил к изменению циркадианной экспрессии гена Per2 (гена часов) в СХЯ, Per1 и Per2 в гиппокампе. Исследование СХЯ через 3 месяца после введения ЛПС выявило усиление экспрессии микроглиальных маркеров CD11b и F4/80 без изменений экспрессии различных нейропептидов, цитокинов и компонентов внутриклеточных сигнальных путей. Эффект введения ЛПС на циркадианный ритм может быть опосредован процессами клеточной гибели, т.к. через 12 ч. после введения ЛПС наблюдался апоптоз клеток СХЯ [43].

Показано выраженную изменчивость тяжести хронических воспалительных заболеваний в течение дня. На модели экспериментального артрита обнаружено, что маркеры воспаления снижены в темновую фазу. Дальнейшие работы доказали, что в воспаленных тканях функционируют активные молекулярные часы и резидентные воспалительные клетки, фибробластоподобные синовиоциты, которые являются возможным источником ритмичных воспалительных сигналов. Воздействие на мышей постоянного освещения нарушало часы в периферических тканях, уменьшая ночное угнетение местного воспаления. Показано также, что основные белки криптохром 1 и 2 снижают уровень воспаления в фибробластоподобных синовиоцитах. Активаторы криптохромов оказывают противовоспалительное действие на клетки человека. 
Таким образом, «биологические часы» активно снижают степень воспалительного процесса в темновую фазу, что может рассматриваться как один из подходов к лечению воспалительных заболеваний [44].

При ревматической полимиалгии (РПМ) ключевые симптомы варьируют в течение суток. Более того, при РПМ уровни мелатонина, некоторых про- и противовоспалительных цитокинов и кортизола повышены в течение дня и подвержены вариабельности. Угнетающее влияние преднизолона показывает, что при РПМ мелатонин стимулирует продукцию цитокинов. Кортизол может снижать продукцию цитокинов и интенсивность симптомов. Стимуляция секреции ИЛ-10 может участвовать в противовоспалительных эффектах преднизолона. Эти результаты поддерживают мнение о пригодности хронотерапии РПМ и необходимости проведения исследований циркадианных вариаций при других воспалительных аутоиммунных заболеваниях [45].

Для циркадианной регуляции развития и специализации иммунных клеток большое значение имеют такие циркадианные осцилляторы, как транскрипционные факторы. Например, NFIL3 (репрессорный ядерный фактор интерлейкина-3, также известный как E4BP4) необходим для развития врожденных лимфоидных клеток (innate lymphoid cells, ILC) - ILC1, ILC2, ILC3 и NK клеток [46]. Такой механизм может участвовать и в развитии ИЛ-17 продуцирующих CD4+ T-хелперов (Th17) [47]. Ритмическая экспрессия, регулируемая клеточными часами, наблюдается и у Toll-подобных рецепторов на эпителиальных клетках кишечника [48]. Параллельные ритмические изменения микробиоты приводят к изменениям Th17 и ILC вблизи барьерных участков [49]. Важным является вопрос об эволюционном основании связи функционирования иммунной системы с циркадианным ритмом. Наиболее обоснованной является точка зрения, что такая связь представляет собой результат естественного отбора для минимизации как затрат организма на обеспечение невосприимчивости/защиты от патогенов, так и восстановление тканей. Таким образом, существует обоюдоострая проблема: с одной стороны обеспечить защиту, с другой - не сделать эту защиту опасной для организма $[50,51]$.
Практически все составляющие врожденного иммунитета проявляют ритмические колебания активности, отличием является то, что их экстремумы наблюдаются в различные временные фазы [52-54]. Циркадианный механизм может контролировать длительность иммунного ответа путем ограничения экспрессии воспалительных генов. Например, делеция BMAL1 или $R E V$-ERBA существенно не нарушает пик ЛПС-индуцированного ответа макрофагов, но уменьшает степень отрицательного экстремума [55].

С учетом циркадианного ритма жизнедеятельности человека весьма логичными выглядят циркадианные ритмы транспорта клеток врожденного иммунитета в очаги воспаления. Нарушения, например, движения моноцитов и нейтрофилов приводят к развитию септических осложнений или чрезмерному повреждению тканей [56, 57]. Поломка циркадианных механизмов регуляции воспаления вследствие эндотоксинемии может приводить к катастрофическим последствиям. Кроме того, может возникать новый профиль генной экспрессии и метаболических ритмов [58]. Ритмические изменения иммунной системы могут задаваться двумя основными механизмами: внешним, в котором принимают участие клетки, «неавтономные» для иммунной системы, и внутренним, который обеспечивается «автономными» клетками иммунной системы [59]. Например, ритмическое высвобождение гемопоэтических стволовых клеток регулируется центральными часами через активность адренэргических нервов. Выброс норадреналина регулирует ритмическую экспрессию и секрецию хемокина CXCL12 стромальными клетками костного мозга [60]. Колебания уровня кортикостероидов обеспечивают появления экстремумов концентраций циркулирующих Т- и В-клеток [61]. Эксперименты показали и четкую зависимость эффективности иммунизации от времени суток [62].

Второй механизм генерирования ритмических колебаний в иммунной системе обеспечивается клеточными автономными циркадианными часами. Например, колебания Ly6Ch моноцитов регулируются клеточной экспрессией BMAL1 [57].

Клеточные часы устанавливают основные колебания генной экспрессии и иммунного 
Огляди

ответа, но факторы внешней среды могут изменять такие колебания иммунных функций двумя основными путями. Во-первых, такие внешние стимулы, как ЛПС могут нарушать фазность, периодичность и амплитуду клеточных циркадианных часов, приводя к потере ритма и сдвигу в сторону патоген-ассоциированного ответа [58, 57]. Во-вторых, основные компоненты клеточных часов могут взаимодействовать с транскрипционными факторами для осуществления ритмических противовоспалительных эффектов. Возможность такого механизма показана для BMAL1-зависимых колебаний экспрессии гена Cxcl5 эпителиальными клетками легких [63].

Эволюционно значимой функцией клеточных часов в клетках, формирующих систему врожденного иммунитета, является угнетение воспалительного ответа. Базальный уровень воспалительного ответа в клетках может регулироваться с помощью циркадианных механизмов двумя путями: 1) основные колебания экспрессии генов цитокинов, опосредованные взаимодействием между BMAL1 и PRC2 (polycomb repressor complex2) [57]; 2) утрата белков CRY, которые выступают потенциальными транскрипционными репрессорами, снижая степень базального и индуцированного воспалительных ответов [64].

Ритмическое воздействие на экспрессию индуцибельных генов может влиять на активность основного провоспалительного фактора NF-kB:

- CLOCK может прямо взаимодействовать с субъединицей p65 NF-kB, усиливая его транскрипционную активность по отношению к провоспалительным генам; при этом BMAL1 может взаимодействовать с CLOCK, вызывая ритмическую репрессию генов воспалительного ответа; отсутствие BMAL1 вызывает дерепрессию вследствие взаимодействия конституционального CLOCK c NF-kB-регулируемыми промоторами [65];

- REV-ERB может опосредовать репрессию путем привлечения $\mathrm{NCoR}$ комплексов HDAC3; REV-ERB репрессируют набор воспалительных генов вследствие ингибирования энхансер-специфической транскрипции; таким образом, циркадианные колебания REV-ERB могут временно ограничивать экспрессию воспалительных генов [66];

- рецептор ГК опосредует репрессивный эффект на провоспалительные цитокины, используя механизмы клеточных часов; ритмическое воздействие BMAL1 участвует в регулировании связывания глюкокортикоидного рецептора с промоторами воспалительных генов, например Cxcl5 [63].

\section{Циркадианная регуляция обмена глюко-} зы, липидов и энергии

Рассмотрим ритмическую регуляцию таких важных процессов, нарушения которых являются компонентами метаболического синдрома, как обмен глюкозы, липидов и энергии. Ранними исследованиями показано существование циркадианных ритмов толерантности к глюкозе [67]. Позднее получены результаты о пике толерантности к глюкозе в утренние часы со снижением в дневное и вечернее время, причем эти эффекты не зависят от длительности голодания [68]. Размах дневных колебаний толерантности к глюкозе очень велик - состояние взрослых пациентов с нормальными показателями утром может быть метаболически эквивалентным предиабетическому состоянию вечером [69].

Изучено влияния изменения светового цикла у мышей 12 ч. : 12 ч. (свет/тьма) до 3 ч. : 3 ч. Ультрадианный цикл приводил к нарушению циркадианного поведенческого цикла, развитию гипергликемии со снижением толерантности к глюкозе, повышению уровней гликированных альбумина и гемоглобина. При ультрадианном ритме увеличивался уровень экспрессии регуляторных генов глюконеогенеза - Pck1, G6pc, HnR4a, FOXO 1/3/4, развивалась гиперхолестеринемия с накоплением холестерина в печени. Однако ультрадианный цикл не изменял уровня воспаления в жировой ткани. Таким образом, доказано существование связи между метаболическими нарушениями и фотопериодическим циклом окружающей среды [70].

Суточные колебания толерантности к глюкозе могут зависеть от многих составляющих, в том числе от отвечаемости $\beta$-клеток, ритмов секреции и клиренса инсулина. Отвечаемость $\beta$-клеток выше утром, чем в другое время суток [71], секреция инсулина в ответ на при- 
ем пищи проявляется более поздним пиком днем [72]. После внутривенного теста толерантности к глюкозе показано, что общая секреция инсулина на 16-51\% выше во вторую половину дня или вечером, чем утром, вследствие продолжительного периода секреции [73]. Клиренс инсулина также подвержен суточным колебаниям, экскреция инсулина заметно ниже утром или вечером [74].

Чувствительность периферических тканей к инсулину также имеет суточные колебания - снижается во второй половине дня [75]. Суточный ритм периферической чувствительности к инсулину обеспечивается как основными внутриклеточными механизмами, так и циркулирующими факторами. Около 15\% транскриптов в клетках скелетных мышц имеют ритмическую модель экспрессии, отражая экспрессию генов, участвующих в обмене глюкозы и липидов [76]. Подкожная жировая ткань также имеет выраженный суточный ритм инсулиновой чувствительности с максимумом днем [77]. Инсулин-опосредованный захват глюкозы наиболее эффективен в утренние часы. Данные инсулиновой чувствительности тканей печени неоднозначны [78]. Важными являются данные об измененных или отсутствующих ритмах толерантности к глюкозе, концентрации глюкозы, периферической чувствительности к инсулину у больных с ожирением и лиц пожилого возраста $[79,75]$. Проведено несколько исследований с изменением фаз день-ночь, которые показали нарушение обмена глюкозы и толерантности к ней [80]. Ожирение также может существенно изменять ритмические процессы толерантности к глюкозе [81]. Таким образом, существуют суточные колебания толерантности к глюкозе со снижением уровня гликемического контроля вечером и ночью.

Циркадианные ритмы отвечаемости $\beta$-клеток, периферической чувствительности к инсулину, клиренса инсулина и эффективности инсулина играют ключевую роль в циркадианном ритме метаболизма глюкозы по сравнению, например, с печеночной чувствительностью к инсулину. Такие ритмы ослаблены у пациентов с диабетом и ожирением, что предполагает причинную роль нарушений циркадианных ритмов в развитии определенных метаболических заболеваний.
Проведено множество исследований, доказывающих существование суточных колебаний уровней холестерина и липопротеинов с различной амплитудой $[82,83]$. Большинство исследователей подтверждают существование суточных ритмов уровня триглицеридов [84]. Данные по ритмам синтеза холестерина остаются несогласованными [85]. Есть предположение, что ритмичность уровня циркулирующих липидов может обеспечиваться циркадианными колебаниями всасывания липидов, их транспортировки и откладывания в организме. Экспериментально доказано, что циркадианная система регулирует всасывание липидов [86]. Исследование, поведенное с участием здоровых женщин с повышенной массой тела, показало, что гены, регулирующие окисление жирных кислот, экспрессируются на 38-82\% слабее вечером, чем утром, а гены, участвующие в липогенезе de nоvo, наоборот, на 51-87\% сильнее [87]. Показано, что среди 15\% плазменных метаболитов, имеющих циркадианные колебания, 80\% относятся к липидным метаболитам [88].

Таким образом, липидная фракция плазмы крови является наиболее регулируемой циркадианной системой, с наибольшим числом пиков утром и в полдень. Такие ритмы наблюдаются вследствие отличий в липидном синтезе, транспорте и депонировании. Существует очень широкая индивидуальная вариабельность времени и силы таких ритмов, возможно, вследствие существования различных циркадианных метаболических фенотипов.

Циркадианный ритм энергетического метаболизма прослеживается на молекулярном и организменном уровнях [89, 90]. Наблюдаемые циркадианные ритмы энергетических затрат связаны с постпрандиальным компонентом. Термический эффект пищи на 44\% выше утром в сравнении с послеполуденными часами и вечером [91]. Постпрандиальное субстратное окисление также может находиться под циркадианным контролем, например, окисление углеводов [71]. Циркадианная система принимает участие и в регуляции аппетита. Показано, что субъекты сообщают о пике аппетита в вечерние часы $[92,93]$. Нужно отметить, что такой регулятор аппетита, как лептин, не проявляет существенных циркадианных ритмов [94], а уровни гормона остро- 
Огляди

го голода грелина не зависят от времени дня, но находятся под воздействием сна, обычного времени приема еды и постпрандиального уровня глюкозы [95].

Нарушения циркадианной регуляции и трансляционные последствия

Суточные ритмы регулируются не только циркадианной системой, но и факторами внешней среды и поведенческими реакциями, в том числе светом, сном, приемом пищи и физической активностью. В настоящее время получены данные, что такие внешние раздражители, как яркий свет ночью, сон в дневное время, прием пищи ночью, не синхронизированы с эндогенным циркадианным ритмом, что приводит к нарушениям метаболизма. Воздействие яркого света днем увеличивает ночную секрецию мелатонина [96]. Снижение уровня освещенности с 5000 до 80 люкс в дневное время ингибирует активность ЖКТ [97].

Терапия ярким светом в дневное время в течение нескольких недель снижала потребность в инсулине у пациентов с диабетом 1-го типа, улучшая гликемический контроль [98]. Утреннее воздействие яркого света (15003000 люкс) на протяжении 3-20 недель может снижать инсулинорезистентность, массу тела и жировую массу, аппетит и повышать набор здоровой массы тела, индуцированный физической нагрузкой, у лиц с ожирением [99]. Важно, что определенные длины световых волн (красные, зеленые или голубые) при недельном воздействии могут ослаблять дисбаланс лептина и грелина, вызванный отсутствием сна [100]. Такие изменения, как освещенность в ночное и вечернее время также связаны с развитием метаболических нарушений. Яркая освещенность (500-600 люкс) вечером увеличивает инсулинорезистентность, повышает постпрандиальные уровни инсулина, глюкозы и глюкагоноподобного пептида 1 [101]. Воздействие яркого света с голубой составляющей (370 люкс) вечером также приводило к повышению постпрандиальной гликемии [102].

Исследование, включавшее около 100000 женщин, показало, что яркий свет в комнате во время сна приводит к повышению индекса массы тела, окружности талии и соотношения окружностей талии и бедер [103]. Воздействие света (18-38 люкс) вечером было связано с большей частотой сахарного диабета [104].
Фазовый сдвиг времени сна, даже если длительность сна остается постоянной, приводит к циркадианным нарушениям.

Ранними исследованиями ночного сдвига рабочего времени (с 9-часовым интервалом) показано неблагоприятное изменение уровней глюкозы и липидов $[105,106]$. Позже было показано, что значительное укорочение длительности сна приводит к уменьшению чувствительности к инсулину [107]. Сон в дневное время снижает энергетические затраты и нарушает субстратное окисление. Показано, что при 9-часовой задержке сна пациенты, спавшие в дневное время, сжигали на 12-16\% меньше калорий [108].

Многими исследованиями показано, что сдвиг времени приема пищи (даже в пределах дневного времени) приводит к метаболическим изменениям. Сдвиг времени ланча с 13.00 на 16.30 повышал уровни глюкозы, снижал затраты энергии и окисление углеводов натощак на 4\%, изменял суточный профиль кортизола [109]. Изменение времени приема пищи на несколько часов существенно изменяло амплитуду и сдвигало ритм синтеза холестерина [110].

Распределение энергетической ценности пищи в течение дня также может приводить к метаболическим изменениям. Показано, что женщины с повышенной массой тела, которые $70 \%$ калорий получали до полудня, за 6 недель теряли на 0,6 кг больше, чем женщины, получавшие 70\% калорий после 16.30 [111]. Потребление основных калорий утром (около $60 \%$ энергии) сопровождается лучшим профилем глюкозы и более высокой чувствительностью к инсулину, чем при приеме вечером (20:30) [112]. Другим исследованием показано, что прием основных калорий (50-54\%) в утреннее время женщинами с избыточной массой тела приводит к большей толерантности к глюкозе [113].

Особенности приема основной части пищи в вечернее время у больных диабетом заключается в сниженной секреции инсулина днем [114]. Снижение секреции инсулина при основном приеме пищи вечером объясняется сниженной продукцией глюкозы печенью у диабетиков [115].

В последние годы показано, что величина воздействия эндогенной циркадианной систе- 
мы на устойчивость к глюкозе и на функции панкреатических $\beta$-клеток превышает воздействие стиля поведения в реализации снижения толерантности к глюкозе от утренних до вечерних часов [71]. Кроме того, у пациентов с предиабетом и с диабетом 2-го типа нарушены циркадианные ритмы температуры тела, частоты сердечных сокращений [116]. Уже сегодня можно говорить о циркадианной этиологии сахарного диабета 2-го типа [117]. Такой взгляд на этиологию сахарного диабета 2-го типа обосновывает хронотерапевтические возможности для нефармакологических методов его лечения [118]. Подобная точка зрения подтверждается и в случае ожирения [119].

\section{Заключение}

Циркадианные механизмы играют важную роль в регуляции обмена веществ, энергетического обмена и иммунных реакций. Изменения образа жизни - такие, как нарушения светового режима, уменьшение амплитуды, температуры окружающей среды, смещение времени приема пищи, распределение энергетической ценности пищи в течение дня приводят к метаболическим нарушениям и развитию низкоинтенсивного системного воспаления. Такие изменения соответствуют понятию метаболического синдрома и могут рассматриваться как «болезнь цивилизации». Молекулярные механизмы циркадианных часов нарушаются при метаболическом синдроме и могут служить мишенью для терапевтических воздействий, в том числе немедикаментозных.

\section{Список использованной литературы}

1. Haller H. Epidermiology and associated risk factors of hyperlipoproteinemia Z Gesamte Inn Med. 1977 Apr 15;32(8):124-8.

2. Pucci G, Alcidi R, Tap L, Battista F, Mattace-Raso F, Schillaci G Sex- and gender-related prevalence, cardiovascular risk and therapeutic approach in metabolic syndrome: A review of the literature. Pharmacol Res. 2017 Jun;120:34-42.

3. Myers J, Kokkinos P, Nyelin E. Physical Activity, Cardiorespiratory Fitness, and the Metabolic Syndrome. Nutrients. $2019 \mathrm{Jul}$ 19;11(7). pii: E1652.

4. Oguntibeju OO. Type 2 diabetes mellitus, oxidative stress and inflammation: examining the links. Int J Physiol Pathophysiol Pharmacol. 2019 Jun 15;11(3):45-63.

5. Кайдашев ИП. Активация NF-kB как молекулярная основа патологического процесса при метаболическом синдроме. Физиологический журнал. 2012, 58(1):93-101. (Kaidashev IP. Activation of NF-kB as the molecular basis of the pathological process in the metabolic syndrome. Physiological Journal. 2012, 58(1):93-101.).

6. Kaidashev IP. Conception for permanent activation of nuclear factor kbeta as molecular basis for metabolic syndrom pathogenesis. Patologicheskaia fiziologiia i eksperimental'naia terapiia. 3 (2013): 65-72.
7. Daan S, Aschoff J. Circadian contribution to survival. In Aschoff J, Daan S, Groos G, editors. Vertebrate Circadian System. Springer-Verlag; Berlin: 1982. pp. 305-21.

8. Pittendrigh CS. Temporal organization: reflections of a Darwinian clock-watcher. Annu Rev Physiol. 1993;55:16-54.

9. Hastings $\mathrm{MH}$, Maywood ES, Brancaccio M. The mammalian circadian timing system and the suprachiasmatic nucleus as its pacemaker biology (Basel). 2019 Mar; 8(1): 13

10. Berson D.M., Dunn F.A., Taka O.M. Phototransduction by retinal ganglion cells that set the circadian clock. Sciense. 2002; Vol. 295 1070-1073.

11. Yamazaki S, Goto M, Menaker M. No evidence for extraocular photoreceptors in the circadian system of the Syrian hamster. J Biol Rhythms. 1999; 14:197-201.

12. Ketema N. Paul, Talib B. Saafir, and Gianluca Tosini The role of retinal photoreceptors in the regulation of circadian rhythms. Rev Endocr Metab Disord. 2009 Dec; 10(4): 271-278.

13. LeGates TA, Fernandez DC, Hattar S. Light as a central modulator of circadian rhythms, sleep and affect. Nat Rev Neurosci. 2014 Jul; 15(7): 443-454.

14. Dibner C, Schibler U, Albrecht U. The mammalian circadian timing system: organization and coordination of central and peripheral clocks. Annu Rev Physiol. 2010;72:517-49.

15. Yoo SH, Yamazaki S, Lowrey PL, Shimomura K, Ko $\mathrm{CH}$ Buhr ED, et al. PERIOD2::LUCIFERASE real-time reporting of circadian dynamics reveals persistent circadian oscillations in mouse peripheral tissues. Proc Natl Acad Sci U S A. 2004 Apr 13;101(15):5339-46

16. Zhang J, Li H, Teng H, Zhang T, Luo Y, Zhao M, et al. Regulation of peripheral clock to oscillation of substance $P$ contributes to circadian inflammatory pain. Anesthesiology. 2012 Jul;117(1):149-60.

17. Grandner MA, Seixas A, Shetty S, Shenoy S. Sleep Duration and Diabetes Risk: Population Trends and Potential Mechanisms. Curr Diab Rep. 2016 Nov;16(11):106.

18. Lowrey PL, Takahashi JS. Mammalian circadian biology: elucidating genome-wide levels of temporal organization. Annu Rev Genomics Hum Genet. 2004;5:407-41.

19. Takahashi JS, Hong HK, Ko CH, McDearmon EL. The genetics of mammalian circadian order and disorder: implications for physiology and disease. Nat Rev Genet. 2008 Oct;9(10):764-75.

20. Hughes ME1, DiTacchio L, Hayes KR, Vollmers C, Pulivarthy S, Baggs JE, et al. Harmonics of circadian gene transcription in mammals. PLoS Genet. 2009 Apr;5(4):e1000442.

21. Le Martelot G, Claudel T, Gatfield D, Schaad O, Kornmann B, Lo Sasso G, et al. REV-ERBalpha participates in circadian SREBP signaling and bile acid homeostasis. PLoS Biol. 2009 Sep;7(9):e1000181.

22. Bass J, Takahashi JS. Circadian integration of metabolism and energetics. Science. 2010 Dec 3;330(6009):1349-54.

23. Cailotto C, La Fleur SE, Van Heijningen C, Wortel J, Kalsbeek A, Feenstra M, et al. The suprachiasmatic nucleus controls the daily variation of plasma glucose via the autonomic output to the liver: are the clock genes involved? Eur J Neurosci. 2005 Nov;22(10):2531-40.

24. Kalsbeek A, Yi CX, La Fleur SE, Fliers E. The hypothalamic clock and its control of glucose homeostasis. Trends Endocrinol Metab. $2010 \mathrm{Jul} ; 21(7): 402-10$.

25. Oster H, Damerow S, Hut RA, Eichele G. Transcriptional profiling in the adrenal gland reveals circadian regulation of hormone biosynthesis genes and nucleosome assembly genes. J Biol Rhythms. 2006 Oct;21(5):350-61

26. Abraham U, Granada AE, Westermark PO, Heine M, Kramer A, Herzel H. Coupling governs entrainment range of circadian clocks. Mol Syst Biol. 2010 Nov 30;6:438.

27. Reinke H, Saini C, Fleury-Olela F, Dibner C, Benjamin IJ, Schibler U. Differential display of DNA-binding proteins reveals heat-shock factor 1 as a circadian transcription factor. Genes Dev. 2008 Feb 1;22(3):331-45.

28. Akerfelt M, Morimoto RI, Sistonen L. Heat shock factors: integrators of cell stress, development and lifespan. Nat Rev Mol Cell Biol. 2010 Aug;11(8):545-55.

29. Yang X, Downes M, Yu RT, Bookout AL, He W, Straume M, et al. Nuclear receptor expression links the circadian clock to metabolism. Cell. 2006 Aug 25;126(4):801-10. 
Огляди

30. Sato TK, Panda S, Miraglia LJ, Reyes TM, Rudic RD, McNamara P, et al. A functional genomics strategy reveals Rora as a component of the mammalian circadian clock. Neuron. 2004 Aug 19;43(4):527-37.

31. Canaple L, Rambaud J, Dkhissi-Benyahya O, Rayet B, Tan NS, Michalik L, et al. Reciprocal regulation of brain and muscle Arntlike protein 1 and peroxisome proliferator-activated receptor alpha defines a novel positive feedback loop in the rodent liver circadian clock. Mol Endocrinol. 2006 Aug:20(8):1715-27.

32. Lin J, Handschin C, Spiegelman BM. Metabolic control through the PGC-1 family of transcription coactivators. Cell Metab. 2005 Jun;1(6):361-70.

33. Liu C., Li S., Liu T., Borjigin J., Lin J. Transcriptional coactivator PGC- $1 \alpha$ integrates the mammalian clock and energy metabolism. Nature, 2007; $447: 477-481$.

34. Rodgers JT1, Lerin C, Haas W, Gygi SP, Spiegelman BM, Puigserver P. Nutrient control of glucose homeostasis through a complex of PGC-1alpha and SIRT1. Nature. 2005 Mar 3;434(7029):113-8.

35. Ramsey KM, Yoshino J, Brace CS, Abrassart D, Kobayashi Y, Marcheva B, et al. Circadian clock feedback cycle through NAMPT-mediated NAD+ biosynthesis. Science. 2009 May 1;324(5927):651-4.

36. Rutter J, Reick M, Wu LC, McKnight SL. Regulation of clock and NPAS2 DNA binding by the redox state of NAD cofactors. Science. 2001 Jul 20;293(5529):510-4

37. Кайдашев ИП. Сиртуины - универсальные регуляторы клеточных функций. Biopolymers and Cell. 2012;28(2):93102. Kaydashev IP. Sirtuins are universal regulators of cellular functions. Biopolymers and Cell. 2012;28(2):93-102.

38. Asher G, Gatfield D, Stratmann M, Reinke H, Dibner C, Kreppel F, et al. SIRT1 regulates circadian clock gene expression through PER2 deacetylation. Cell. 2008 Jul 25;134(2):317-28.

39. Nakahata Y, Kaluzova M, Grimaldi B, Sahar S, Hirayama J Chen $\mathrm{D}$, et al. The NAD+-dependent deacetylase SIRT1 modulates CLOCK-mediated chromatin remodeling and circadian control. Cell. 2008 Jul 25;134(2):329-40.

40. Guerreno-Vargas N.N., Guzman-Ruiz M., Fuentes R., Garsia J., et al. Shift work in rats results in increased inflammatory response after lipopolysaccaride administration: role for food consumption. J Biol. Rhytms. 2015;30(4):318-330.

41. Guerro-Vargas NN, Salgado-Delgado R., Basualdo MdelC Garsia J. et al. Reciprocal interaction between the suprachiasmatic nucleus and the immune system tunes down the inflammatory response to lipopolysaccharide. Neuroimmunol. 2014; 273(12):22-30.

42. Oyama Y., Iwasaka H., Koga H., Shihgu C., Matsumoto S., Noguchi T. Uncoupling of peripheral and master clock gene rythms by reversed leads to an exacerbated inflammatory response after polymicrobial sepsis in mice. Schok. 2014; 41(3):214-221.

43. O'Callaghan EK, Anderson ST, Moynagh PN, Coogan AN. Longlasting effects of sepsis on circadian rhythms in the mouse. PLoS One. 2012;7(10):e47087.

44. Hand LE, Hopwood TW, Dickson SH, Walker AL, Loudon AS, Ray DW, et al. The circadian clock regulates inflammatory arthritis. FASEB J. 2016 Nov;30(11):3759-3770.

45. Galbo H. et.al. Circadian variations in clinical symptoms and concentrations of inflammatory cytokines, melatonin, and cortisol in polymyalgia rheumatica before and during prednisolone treatment: a controlled, observational, clinical experimental study. Arthritis Research \& Therapy. 2016;18:174.

46. Seillet C, Rankin LC, Groom JR, Mielke LA, Tellier J, Chopin M, et al. Nfil3 is required for the development of all innate lymphoid cell subsets. J Exp Med. 2014 Aug 25;211(9):1733-40.

47. Yu X, Rollins D, Ruhn KA, Stubblefield JJ, Green CB, Kashiwada M, et al. TH17 cell differentiation is regulated by the circadian clock. Science. 2013 Nov. 8;342(6159):727-30.

48. Mukherji A, Kobiita A, Ye T, Chambon P. Homeostasis in intestinal epithelium is orchestrated by the circadian clock and microbiota cues transduced by TLRs. Cell. 2013 May 9;153(4):812-27.

49. Thaiss CA, Zeevi D, Levy M, Zilberman-Schapira G, Suez J Tengeler AC, et al. Transkingdom control of microbiota diurnal oscillations promotes metabolic homeostasis. Cell. 2014 Oct 23;159(3):514-29.
50. Okin D, Medzhitov R. Evolution of inflammatory diseases. Curr Biol. 2012 Sep 11;22(17):R733-40

51. Stearns S.C. Medzhitov R. Evolutionary medicine. Sinauer Associates, Inc. Publishers; Sunderland, Massachussetts: 2016. p. xix. p.306.

52. Curtis AM, Bellet MM, Sassone-Corsi P, O'Neill LA. Circadian clock proteins and immunity. Immunity. 2014 Feb 20;40(2):178-86.

53. Casanova-Acebes M, Pitaval C, Weiss LA, Nombela-Arrieta C, Chèvre R, A-González $\mathrm{N}$, et al. Rhythmic modulation of the hematopoietic niche through neutrophil clearance. Cell. 2013 May 23;153(5):1025-35.

54. Silver AC, Arjona A, Walker WE, Fikrig E. The circadian clock controls toll-like receptor 9-mediated innate and adaptive immunity. Immunity. 2012 Feb 24;36(2):251-61.

55. Gibbs JE, Blaikley J, Beesley S, Matthews L, Simpson KD, Boyce $\mathrm{SH}$, et al. The nuclear receptor REV-ERB $\alpha$ mediates circadian regulation of innate immunity through selective regulation of inflammatory cytokines. Proc Natl Acad Sci U S A 2012 Jan 10;109(2):582-7.

56. Scheiermann C, Kunisaki Y, Lucas D, Chow A, Jang JE, Zhang D, et al. Adrenergic nerves govern circadian leukocyte recruitment to tissues. Immunity. 2012 Aug 24;37(2):290-301.

57. Nguyen KD, Fentress SJ, Qiu Y, Yun K, Cox JS, Chawla A Circadian gene Bmal1 regulates diurnal oscillations of Ly6C(hi) inflammatory monocytes. Science. 2013 Sep 27;341(6153):1483-8.

58. Haspel JA, Chettimada S, Shaik RS, Chu JH, Raby BA, Cernadas M, et al. Circadian rhythm reprogramming during lung inflammation. Nat Commun. 2014 Sep 11;5:4753.

59. Man K, Loudon A, Chawla A. Immunity around the clock. Science. 2016 Nov 25;354(6315):999-1003

60. Méndez-Ferrer S, Lucas D, Battista M, Frenette PS. Haematopoietic stem cell release is regulated by circadian oscillations. Nature. 2008 Mar 27;452(7186):442-7.

61. Kawate T, Abo T, Hinuma S, Kumagai K. Studies of the bioperiodicity of the immune response. II. Co-variations of murine T and B cells and a role of corticosteroid. J Immunol. 1981 Apr;126(4):1364-7.

62. Labrecque N, Cermakian N. Circadian Clocks in the Immune System. J Biol Rhythms. 2015 Aug;30(4):277-90

63. Gibbs J, Ince L, Matthews L, Mei J, Bell T, Yang N, et al. An epithelial circadian clock controls pulmonary inflammation and glucocorticoid action. Nat Med. 2014 Aug;20(8):919-26.

64. Narasimamurthy R, Hatori M, Nayak SK, Liu F, Panda S, Verma IM. Circadian clock protein cryptochrome regulates the expression of proinflammatory cytokines. Proc Natl Acad Sci USA. 2012 Jul 31;109(31):12662-7.

65. Spengler ML, Kuropatwinski KK, Comas M, Gasparian AV, Fedtsova N, Gleiberman AS, et al. Core circadian protein CLOCK is a positive regulator of NF- $\mathrm{KB}$-mediated transcription. Proc Natl Acad Sci U S A. 2012 Sep 11:109(37):E2457-65.

66. Eichenfield DZ, Troutman TD, Link VM, Lam MT, Cho H, Gosselin D, et al. Tissue damage drives co-localization of NF$\kappa \mathrm{B}, \mathrm{Smad} 3$, and Nrf2 to direct Rev-erb sensitive wound repair in mouse macrophages. Elife. $2016 \mathrm{Jul}$ 27;5.

67. Walsh C. H., Wright A. D. Diurnal patterns of oral glucose tolerance in diabetics. Postgrad Med J. 1975 Mar; 51(593):169-172.

68. Hulmán A, Færch K, Vistisen D, Karsai J, Nyári TA, et al. Effect of time of day and fasting duration on measures of glycaemia: analysis from the Whitehall II Study. Diabetologia. 2013 Feb;56(2):294-7.

69. Carroll KF, Nestel PJ. Diurnal variation in glucose tolerance and in insulin secretion in man. Diabetes. 1973 May;22(5):333-48.

70. Oishi K, Itoh N. Disrupted daily light-dark cycle induces the expression of hepatic gluconeogenic regulatory genes and hyperglycemia with glucose intolerance in mice. Biochem Biophys Res Commun. 2013 Mar 1;432(1):111-5.

71. Morris CJ, Yang JN, Garcia JI, Myers S, Bozzi I, Wang W, et al Endogenous circadian system and circadian misalignment impact glucose tolerance via separate mechanisms in humans. Proc Natl Acad Sci U S A. 2015 Apr 28;112(17): E2225-34

72. Boden G, Ruiz J, Urbain JL, Chen X. Evidence for a circadian rhythm of insulin secretion. Am J Physiol. 1996 Aug;271(2 Pt 1): E246-52.

73. Calles-Escandon J, Jaspan J, Robbins DC. Postprandial oscillatory patterns of blood glucose and insulin in NIDDM. Abnormal diurnal insulin secretion patterns and glucose homeostasis independent of obesity. Diabetes Care. 1989 Nov-Dec;12(10):709-14. 
74. Saad A, Dalla Man C, Nandy DK, Levine JA, Bharucha AE Rizza RA, et al. Diurnal pattern to insulin secretion and insulin action in healthy individuals. Diabetes. 2012 Nov;61(11):2691-700.

75. Lee A, Ader M, Bray GA, Bergman RN. Diurnal variation in glucose tolerance. Cyclic suppression of insulin action and insulin secretion in normal-weight, but not obese, subjects. Diabetes. 1992 Jun;41(6):750-9.

76. Hansen J, Timmers S, Moonen-Kornips E, Duez H, Staels B, Hesselink MK, et al. Synchronized human skeletal myotubes of lean, obese and type 2 diabetic patients maintain circadian oscillation of clock genes. Sci Rep. 2016 Oct 19;6:35047.

77. Carrasco-Benso MP, Rivero-Gutierrez B, Lopez-Minguez J, Anzola A, Diez-Noguera A, Madrid JA, et al. Human adipose tissue expresses intrinsic circadian rhythm in insulin sensitivity. FASEB J. 2016 Sep;30(9):3117-23.

78. Verrillo A, De Teresa A, Martino C, Di Chiara G, Pinto M Verrillo L, et al. Differential roles of splanchnic and peripheral tissues in determining diurnal fluctuation of glucose tolerance. Am J Physiol. 1989 Oct;257(4 Pt 1): E459-65

79. Pisu E, Diana A, Lombardi A, Cassader M, Pagano G. Diurnal variations in insulin secretion and insulin sensitivity in aged subjects. Acta Diabetol Lat. 1980 Apr-Jun;17(2):153-60.

80. Van Cauter E, Blackman JD, Roland D, Spire JP, Refetoff S, Polonsky KS. Modulation of glucose regulation and insulin secretion by circadian rhythmicity and sleep. J Clin Invest. 1991 Sep;88(3):934-42.

81. Van Cauter EV, Polonsky KS, Blackman JD, Roland D, Sturis J, Byrne MM, et al. Abnormal temporal patterns of glucose tolerance in obesity: relationship to sleep-related growth hormone secretion and circadian cortisol rhythmicity. J Clin Endocrinol Metab. 1994 Dec;79(6):1797-805.

82. Romon M, Le Fur C, Lebel P, Edmé JL, Fruchart JC, Dallongeville J. Circadian variation of postprandial lipemia. Am J Clin Nutr. 1997 Apr;65(4):934-40

83. Van Kerkhof LW, Van Dycke KC, Jansen EH, Beekhof PK, van Oostrom CT, Ruskovska T, et al. Diurnal Variation of Hormonal and Lipid Biomarkers in a Molecular Epidemiology-Like Setting. PLoS One. 2015 Aug 18;10(8): e0135652.

84. Sennels HP, Jørgensen HL, Fahrenkrug J. Diurnal changes of biochemical metabolic markers in healthy young males - the Bispebjerg study of diurnal variations. Scand J Clin Lab Invest. 2015;75(8):686-92.

85. Miettinen TA. Diurnal variation of cholesterol precursors squalene and methyl sterols in human plasma lipoproteins. J Lipid Res. 1982 Mar;23(3):466-73.

86. Hussain MM, Pan X. Circadian regulators of intestinal lipid absorption. J Lipid Res. 2015 Apr;56(4):761-70.

87. Yoshino J, Almeda-Valdes P, Patterson BW, Okunade AL, Imai S, Mittendorfer B, et al. Diurnal variation in insulin sensitivity of glucose metabolism is associated with diurnal variations in wholebody and cellular fatty acid metabolism in metabolically normal women. J Clin Endocrinol Metab. 2014 Sep;99(9): E1666-70.

88. Dallmann R, Viola AU, Tarokh L, Cajochen C, Brown SA. The human circadian metabolome. Proc Natl Acad Sci U S A. 2012 Feb 14;109(7):2625-9.

89. McGinnis GR, Young ME. Circadian regulation of metabolic homeostasis: causes and consequences. Nat Sci Sleep. 2016 May 27:8:163-80.

90. Kumar Jha P, Challet E, Kalsbeek A. Circadian rhythms in glucose and lipid metabolism in nocturnal and diurnal mammals. Mol Cell Endocrinol. 2015 Dec 15;418 Pt 1:74-88

91. Zwiauer KF, Mueller T, Widhalm K. Effect of daytime on resting energy expenditure and thermic effect of food in obese adolescents. J Am Coll Nutr. 1992 Jun;11(3):267-71

92. Wehrens SMT, Christou S, Isherwood C, Middleton B, Gibbs MA, Archer SN, et al. Meal Timing Regulates the Human Circadian System. Curr Biol. 2017 Jun 19;27(12):1768-1775.

93. Scheer FA, Morris CJ, Shea SA. The internal circadian clock increases hunger and appetite in the evening independent of food intake and other behaviors. Obesity (Silver Spring). 2013 Mar;21(3):421-3.

94. Scheer FA, Hilton MF, Mantzoros CS, Shea SA. Adverse metabolic and cardiovascular consequences of circadian misalignment. Proc Natl Acad Sci U S A. 2009 Mar 17;106(11):4453-8.
95. Natalucci G, Riedl S, Gleiss A, Zidek T, Frisch H. Spontaneous 24-h ghrelin secretion pattern in fasting subjects: maintenance of a meal-related pattern. Eur J Endocrinol. 2005 Jun;152(6):845-50.

96. Mishima K, Okawa M, Shimizu T, Hishikawa Y. Diminished melatonin secretion in the elderly caused by insufficient environmental illumination. J Clin Endocrinol Metab. 2001 Jan;86(1):129-34.

97. Sone Y, Hyun KJ, Nishimura S, Lee YA, Tokura H. Effects of dim or bright-light exposure during the daytime on human gastrointestinal activity. Chronobiol Int. 2003 Jan;20(1):123-33.

98. Nieuwenhuis RF, Spooren PF, Tilanus JJ. Less need for insulin, a surprising effect of phototherapy in insulin-dependent diabetes mellitus. Tijdschr Psychiatr. 2009;51(9):693-7.

99. Sene-Fiorese M, Duarte FO, de Aquino Junior AE, Campos RM, Masquio DC, Tock L, et al. The potential of phototherapy to reduce body fat, insulin resistance and «metabolic inflexibility» related to obesity in women undergoing weight loss treatment. Lasers Surg Med. 2015 Oct;47(8):634-42.

100. Figueiro MG, Plitnick B, Rea MS. Light modulates leptin and ghrelin in sleep-restricted adults. Int $\mathrm{J}$ Endocrinol. 2012;2012:530726.

101. Gil-Lozano M, Hunter PM, Behan LA, Gladanac B, Casper RF, Brubaker PL. Short-term sleep deprivation with nocturnal light exposure alters time-dependent glucagon-like peptide-1 and insulin secretion in male volunteers. Am J Physiol Endocrinol Metab. 2016 Jan 1;310(1): E41-50.

102. Cheung IN, Zee PC, Shalman D, Malkani RG, Kang J, Reid KJ. Morning and Evening Blue-Enriched Light Exposure Alters Metabolic Function in Normal Weight Adults. PLoS One. 2016 May 18;11(5): e0155601.

103. McFadden E, Jones ME, Schoemaker MJ, Ashworth A, Swerdlow AJ. The relationship between obesity and exposure to light at night: cross-sectional analyses of over 100,000 women in the Breakthrough Generations Study. Am J Epidemiol. 2014 Aug 1;180(3):245-50

104. Obayashi K, Saeki K, Iwamoto J, Ikada Y, Kurumatani N. Independent associations of exposure to evening light and nocturnal urinary melatonin excretion with diabetes in the elderly. Chronobiol Int. 2014 Apr;31(3):394-400.

105. Hampton SM, Morgan LM, Lawrence N, Anastasiadou T, Norris F, Deacon S, et al. Postprandial hormone and metabolic responses in simulated shift work. J Endocrinol. 1996 Nov;151(2):259-67.

106. Ribeiro DC, Hampton SM, Morgan L, Deacon S, Arendt J. Altered postprandial hormone and metabolic responses in a simulated shift work environment. J Endocrinol. 1998 Sep;158(3):305-10.

107. Leproult R, Holmbäck U, Van Cauter E. Circadian misalignment augments markers of insulin resistance and inflammation, independently of sleep loss. Diabetes. 2014 Jun;63(6):1860-9.

108. McHill AW, Melanson EL, Higgins J, Connick E, Moehlman TM, Stothard ER, et al. Impact of circadian misalignment on energy metabolism during simulated nightshift work. Proc Natl Acad Sci USA. 2014 Dec 2;111(48):17302-7.

109. Bandín C, Scheer FA, Luque AJ, Ávila-Gandía V, Zamora S, Madrid JA, et al. Meal timing affects glucose tolerance, substrate oxidation and circadian-related variables: A randomized, crossover trial. Int J Obes (Lond). 2015 May;39(5):828-33.

110. Cella LK, Van Cauter E, Schoeller DA. Effect of meal timing on diurnal rhythm of human cholesterol synthesis. Am J Physiol. 1995 Nov;269(5 Pt 1): E878-83.

111. Keim NL, Van Loan MD, Horn WF, Barbieri TF, Mayclin PL. Weight loss is greater with consumption of large morning meals and fat-free mass is preserved with large evening meals in women on a controlled weight reduction regimen. J Nutr. 1997 Jan;127(1):75-82.

112. Morgan LM, Shi JW, Hampton SM, Frost G. Effect of meal timing and glycaemic index on glucose control and insulin secretion in healthy volunteers. Br J Nutr. 2012 Oct;108(7):1286-91.

113. Jakubowicz D, Barnea M, Wainstein J, Froy O. Effects of caloric intake timing on insulin resistance and hyperandrogenism in lean women with polycystic ovary syndrome. Clin Sci (Lond). 2013 Nov;125(9):423-32.

114. Beebe CA, Van Cauter E, Shapiro ET, Tillil H, Lyons R, Rubenstein AH, Polonsky KS. Effect of temporal distribution of calories on diurnal patterns of glucose levels and insulin secretion in NIDDM. Diabetes Care. 1990 Jul;13(7):748-55. 


\section{Огляди}

115. Boden G, Chen X, Urbain JL. Evidence for a circadian rhythm of insulin sensitivity in patients with NIDDM caused by cyclic changes in hepatic glucose production. Diabetes. 1996 Aug;45(8):1044-50.

116. Gubin DG, Nelaeva AA, Uzhakova AE, Hasanova YV, Cornelissen G, Weinert D. Disrupted circadian rhythms of body temperature, heart rate and fasting blood glucose in prediabetes and type 2 diabetes mellitus. Chronobiol Int. 2017;34(8):1136-1148.

117. Javeed N, Matveyenko AV. Circadian Etiology of Type 2 Diabetes Mellitus. Physiology (Bethesda). 2018 Mar 1;33(2):138-150.

118. Karthikeyan R, Marimuthu G, Spence DW, Pandi-Perumal SR, BaHammam AS, Brown GM, Cardinali DP. Should we listen to our clock to prevent type 2 diabetes mellitus? Diabetes Res Clin Pract. 2014 Nov;106(2):182-90.

119. Leslie M. Circadian rhythms. Sleep study suggests triggers for diabetes and obesity. Science. 2012 Apr 13;336(6078):143.

(Надійшла до редакції 24.02.2020р.)

\section{Роль молекулярного годинника циркадіанних ритмів у патогенезі метаболічного синдрому}

\section{І.П. Кайдашев}

Українська медична стоматологічна академія, м. Полтава

Резюме. Метаболічний синдром об'єднує порушення метаболізму, що призводять до збільшення маси тіла із супутнім системним запаленням. Важливим $є$ виявлення патогенетичних зв'язків між порушеннями обміну глюкози, ліпідів, окислювальним стресом, запаленням і збільшенням маси тіла та молекулярними механізмами циркадіанного годинника. В огляді наведено сучасні дані щодо забезпечення циркадіанних ритмів (ЦР) за участю центрального та периферичних пейсмекерів, їх молекулярних складових та й іє рархії. Головні чинники порушень ЦР - зміна тривалості циклів «світло-темрява», порушення світлового режиму, тривалості сну, вживання їжі в другій половині дня. Порушення ЦР викликає зниження толерантності до глюкози, підвищення інсулінорезистентності тканин, порушує всмоктування, транспортування та депонування ліпідів в організмі та призводить до накопичення надлишків енергії. Такі метаболічні порушення відбуваються паралельно 3 підвищенням рівня системного запалення: продукування прозапальних хемо- і цитокінів, активації імунних клітин.
Зроблено висновок, що порушення ЦР призводить до метаболічних порушень і системного запалення, що відповідає поняттю «метаболічного синдрому».

Ключові слова: метаболічний синдром, молекулярний годинник, запалення, обмін вуглеводів.

\section{The role of the molecular clock of circadian rhythms in the pathogenesis of metabolic syndrome}

\section{I.P. Kaidashev \\ Ukrainian Medical Stomatological Academy, Poltava}

Abstract. Metabolic syndrome combines metabolic disorders that lead to weight gain with concomitant systemic inflammation. It is important to identify the pathogenetic links between the disrupted metabolism of glucose, lipids, oxidative stress, inflammation and weight gain, as well as the molecular mechanisms of the circadian clock. The review provides the contemporary data on ensuring the circadian rhythms (CR), involving central and peripheral pacemakers, their molecular component and hierarchy. The main factors in the $C R$ disruption are changes in the duration of light-dark cycles, disturbance of light regime, duration of sleep, food intake in the afternoon. Disruption of the CR causes a decreased glucose tolerance, an increased insulin resistance of tissues, and impairs absorption, transport and deposition of lipids in the body, leading to the accumulation of excess energy. Such metabolic disorders occur in parallel with an increased level of systemic inflammation: the production of proinflammatory chemo- and cytokines, activation of immune cells.

It has been concluded that the CR disorder leads to metabolic disruptions and systemic inflammation, which corresponds to the concept of metabolic syndrome.

Keywords: metabolic syndrome, molecular clock, inflammation, carbohydrates metabolism. 